\title{
Objective Assessment of the Severity of Patients Suffering from Fall from Height with Combined Injuries of the Abdominal Parenchymal Organs
}

\author{
Abdukhakim Khadjibaev, PhD, ScD; Pulat Sultanov* \\ Republican Scientific Center of Emergency Medicine \\ Tashkent, Uzbekistan
}

\begin{abstract}
In recent years, fall from a height (FFH) has been a relatively frequent cause of injury and death in the urban environment. The purpose of this study was to optimize the risk stratification of FFH victims with combined injuries of the abdominal organs by using Injury Severity Score (ISS) scale. The study included 111 patients (aged between 15 and 80 years) injured by FFH. All the falls were accidental and occurred mainly among males (82\%). The height of the fall ranged from 2 to 5 meters. Combined injuries were found in 98 patients and isolated injuries in 13 patients. The combination of the 6 injured body regions was identified in 5 patients, 5 regions in 17, 4 in 35, 3 in 23, and 2 in 18. The abdomen trauma was most commonly associated with the following injured body regions: head and neck-chest-extremities and pelvis (13.3\%), head and neck-chest-extremities (12.2\%), and head and neck-chest-pelvis (9.2\%). Among the combined injuries of the abdomen, ruptures of parenchymal organs (liver, spleen and kidneys) were predominant. To assess the severity of the injury, the ISS scale was applied. The injuries of abdominal parenchymal organs were evaluated according to the AAST (American Association for the Surgery of Trauma) classification. Comparative analysis of the assessment of the severity of a patient's condition according to the traditional scale and the ISS scale showed that the ISS scale promotes the active and timely detection of the extremely severe and terminal condition in patients with injuries due to FFH with combined trauma of the abdominal organs. Objective assessment of the severity of trauma and the dominant injury region allows determining the optimal treatment algorithm and predicting the outcome of the injury.
\end{abstract}

Keywords: fall from a height; combined injuries; abdominal trauma, Injury Severity Score; severity of a patient's condition.

\section{Introduction}

In recent years, fall from a height (FFH) has been a relatively frequent cause of injury and death in the urban environment $[1,2]$. Injuries due to FFH are characterized by severe associated injuries of different organs and systems, including severe abdominal trauma. The high frequency and prevalence of multiple injuries of abdominal organs determine the importance of this problem in emergency medicine [3-7]. The combination of abdominal trauma with injuries of other anatomical regions complicates the severity of injured patients and diagnosis of injuries that worsen the prognosis $[4,5,8]$. At equal severity of the injury and pathophysiological changes,

*Corresponding author: Pulat Sultanov. Republican Scientific Center of Emergency Medicine Tashkent, Uzbekistan E-mail: sultanovp@bk.ru the severity of a patient's condition depends on the functional reserves and adaptive capacities of the organism. In this regard, an objective assessment of the severity of a patient's condition presents certain difficulties [3,9-11].

A traditional classification of the severity of a patient's condition lacks clear criteria and unity of interpretation [12]. This classification is widely used in everyday practice and describes a patient's condition as satisfactory, moderate, severe, extremely severe or terminal. To assess the severity of a patient's condition, various scales and indices are suggested, which are based on a score of the clinical symptoms or laboratory indicators [11-13]. An objective assessment of a patient's condition at admission to hospital allows maximum avoidance of diagnostic and tactical mistakes, and improves the accuracy of decisions $[9,14,15]$. At the same time, a number of authors have noted that the integrated systems are not reliable and have suggested possible ways to improve them. 
Thus, the MFS-AC scale (MFS - military field surgery, A - admission, C - condition) developed by EK Gumanenko et al [16] is focused on the clinical signs. Calculations are based on a score of 12 of the most important and easily identifiable clinical signs. Among these are the color of the skin and condition of the respiratory system, central nervous system, circulatory system, and gastrointestinal tract, as well as the magnitude of blood loss. In many countries, an assessment of the severity of a patient's condition at admission is performed by using the ISS (Injury Severity Score). This scale best meets the requirements of emergency surgery and scientific analysis. The ISS scale is easy to use and requires no additional equipment. Among anatomical severity scores, the ISS scale created by Baker et al. in 1974 [10], has been considered for over 40 years to be the gold standard to classify trauma injuries, both blunt and penetrating $[3,4,10,11,16]$. The ISS is based upon the Abbreviated Injury Scale (AIS). AIS is one of the most common anatomic scales for traumatic injuries. The first version of the scale was published in 1969 [17] with major updates in 1976, 1980, 1985, 1990, 1998, 2005, and 2008. The AIS is a consensus-derived, anatomically based system of grading injuries on an ordinal scale ranging from 1 (minor injury) to 6 (lethal injury) [18]. The ISS is obtained by summing the square value of the 3 highest AIS scores, identifying the severity of patients and enabling stratification of them. Six body regions are defined, as follows: head and neck, face, chest, abdomen, extremities (including pelvis), and external structures. Only one injury per body region is allowed. The ISS ranges from 1 to 75 , and an ISS of 75 is assigned to anyone with AIS of 6 .

Despite the numerous advantages of the ISS, it has some limitations $[19,20]$. The most obvious limitation is its inability to account for multiple injuries to the same body region; therefore, the ISS scale is rarely used to assess the severity of injury in victims of FFH with combined injuries of the abdominal organs.

The purpose of this study is to optimize the risk stratification of FFH victims with combined injuries of the abdominal organs by using ISS.

\section{Material and Methods}

The study included 111 patients (aged between 15 and 80 years, mean age $34.46 \pm 5.92 \mathrm{yrs}$ ) injured by FFH. All the falls were accidental and occurred mainly among males $(82 \%)$. The height of the fall ranged from 2 to 5 meters. All patients were treated in the Republican Scientific Center of Emergency Medicine (RSCEM) between 2010 and 2013. The study was approved by the RSCEM Ethics Committee. Written informed consent was obtained from each patient.

Combined injuries were found in 98 patients and isolated injuries in 13 patients. The combination of the 6 injured body regions was identified in 5 patients, 5 regions in 17, 4 in 35, 3 in 23 , and 2 in 18 . The abdomen trauma was most commonly associated with the following injured body regions: head and neck-chest-extremities and pelvis (13.3\%), head and neckchest-extremities (12.2\%), head and neck-chest-pelvis $(9.2 \%)$, head and neck-chest (9.2\%), head and neck-chest - extremities and pelvis-spine $(5.1 \%)$, chest $(5.1 \%)$, head and neck-chestspine $(4.1 \%)$, pelvis $(4.1 \%)$, head and neck-extremities and pelvis $(3.1 \%)$, head and neck- extremities $(3.1 \%)$, chest-pelvis -spine $(3.1 \%)$, and chest-spine $(3.1 \%)$. The remaining 25 percent included other rare combinations.

Among the combined injuries of the abdomen, ruptures of parenchymal organs were predominant (Table 1). Spleen injuries were observed in $30(27 \%)$ of cases, liver injuries in $28(25.2 \%)$ cases, and combined injuries of spleen and liver were observed in $6(5.4 \%)$ cases. Isolated injuries of spleen and liver occurred in $17(15.3 \%)$ and $14(12.6 \%)$ cases, respectively. In other cases, the traumatic injuries of abdominal parenchymal organs (liver, spleen and kidneys) had a multiple character. Traumatic injuries of hollow organs were observed in $12(12.8 \%)$ patients. Retroperitoneal hematoma occurred in $19(17.1 \%)$ cases.

To assess the severity of the injury, the ISS scale was applied [10]. The injuries of abdominal parenchymal organs were evaluated according to the AAST (American Association for the Surgery of Trauma) classification [21]. According to this classification, the following injuries were diagnosed: Grade I splenic injury was in 1(3\%) patient, Grade II in 2(7\%) patients, Grade III in $11(37 \%)$ patients, and Grade IV in $16(53 \%)$ patients. Grade V splenic injury was not identified. Grade I hepatic injury was observed in 5 patients, Grade II in 8 patients, grade III in 11 patients, and grade IV in 4 patients. Grade V hepatic injury was not identified. The distribution of patients into groups depending on the degree of injury of parenchymal organs was carried out based on the data of clinical-instrumental methods of investigation and intraoperative findings. An ISS score was obtained by summing the square value of the 3 highest AIS scores: from 1 (minor injury) to 6 (lethal injury). Major trauma was defined using an ISS threshold of 13.

Statistical analysis was performed using the SPSS 10.0 software package (SPSS, Chicago, IL, USA). The mean (M) and standard error of the mean (SEM) were deduced. Student's unpaired and paired t-tests were used to compare two groups for data with normal distribution. We also used the Chi-square test to compare observed data. $P$ values of $<0.05$ were considered statistically significant.

\section{Results and Discussion}

Assessment of the severity of a patient's condition according to the traditional scale and the ISS scale is presented in Table 2. The severity of the injuries depended on the combination of the anatomical regions involved. In our studies, the isolated injuries of the abdominal organs were mainly assessed as satisfactory and moderate severity; when the involvement of other anatomical regions increased, the number of patients with a severe, extremely severe and terminal condition also increased. The maximum score, ISS of 75 , was assigned to 4 patients; 2 of them died within the first hour. The severity of these patients with traumatic cerebral injury had an AIS score of 6. The remaining 2 patients died on the first day; in these patients, we found a dominant combination of three anatomical regions with AIS of 5, which resulted in an ISS of 75. 
Table 1.

The frequency of the abdomen trauma in FFH victims

\begin{tabular}{|c|c|c|c|}
\hline № & Combined Injuries of the Abdominal Organs & Abs & $\%$ \\
\hline 1. & Contusion and hematoma of anterior abdominal wall & 41 & 36.9 \\
\hline 2. & Injuries of internal organs. Hemoperitoneum. & 3 & 2.7 \\
\hline 3. & Splenic capsular tear & 1 & 0.9 \\
\hline 4. & Splenic capsular tear + hepatic hematoma & 1 & 0.9 \\
\hline 5. & Splenic rupture & 17 & 15.3 \\
\hline 6. & Splenic rupture + retroperitoneal hematoma & 1 & 0.9 \\
\hline 7. & Splenic rupture + kidney rupture & 1 & 0.9 \\
\hline 8. & Splenic rupture + kidney rupture + retroperitoneal hematoma & 1 & 0.9 \\
\hline 9. & Splenic rupture + kidney rupture + rupture of the pancreatic capsule + retroperitoneal hematoma & 1 & 0.9 \\
\hline 10. & Splenic rupture + hepatic rupture/hepatic capsular tear & 2 & 1.8 \\
\hline 11. & Splenic rupture + hepatic rupture + retroperitoneal hematoma & 1 & 0.9 \\
\hline 12. & Splenic rupture + hepatic rupture + rupture of the small-intestinal mesentery + retroperitoneal hematoma & 1 & 0.9 \\
\hline 13. & Splenic rupture + gallbladder avulsion + retroperitoneal hematoma & 1 & 0.9 \\
\hline 14. & Splenic rupture + bladder rupture + deserosation of colon + retroperitoneal hematoma & 1 & 0.9 \\
\hline 15. & Splenic rupture + ovarian apoplexy + deserosation of colon + retroperitoneal hematoma & 1 & 0.9 \\
\hline 16. & Hepatic hematoma & 2 & 1.8 \\
\hline 17. & Hepatic capsular tear & 2 & 1.8 \\
\hline 18. & Hepatic rupture & 10 & 9.0 \\
\hline 19. & Hepatic rupture + retroperitoneal hematoma & 2 & 1.8 \\
\hline 20. & Hepatic rupture + contusion of the pancreatic head & 1 & 0.9 \\
\hline 21. & Hepatic rupture + contusion of the pancreatic head + hepatoduodenal ligament hematoma & 1 & 0.9 \\
\hline 22. & Hepatic rupture + ovarian apoplexy + deserosation of colon & 1 & 0.9 \\
\hline 23. & Hepatic rupture + rupture of the small intestine + diffuse serofibrinous peritonitis & 1 & 0.9 \\
\hline 24. & Hepatic rupture + rupture of the small intestine + tear in the stomach wall + diffuse serofibrinous peritonitis & 1 & 0.9 \\
\hline 25. & Kidney rupture + colon rupture + diffuse serofibrinous peritonitis & 1 & 0.9 \\
\hline 26. & Rupture of the small Intestine + diffuse serofibrinous peritonitis & 2 & 1.8 \\
\hline 27. & $\begin{array}{l}\text { Rupture of the small Intestine + rupture of the small-intestinal mesentery + rupture of the pancreatic capsule }+ \\
\text { hepatic capsular tear }+ \text { tear in the stomach wall }+ \text { deserosation of colon }+ \text { diffuse serofibrinous peritonitis }\end{array}$ & 1 & 0.9 \\
\hline 28. & Rupture of the small-intestinal mesentery + retroperitoneal hematoma & 1 & 0.9 \\
\hline 29. & Rupture of the small-intestinal mesentery + contusion of the pancreatic head + retroperitoneal hematoma & 1 & 0.9 \\
\hline 30. & Gastrointestinal ligament hematoma + retroperitoneal hematoma & 1 & 0.9 \\
\hline 31. & Retroperitoneal hematoma & 6 & 5.4 \\
\hline 32. & Penetrating abdominal trauma without injuries of abdominal organs & 1 & 0.9 \\
\hline 33. & Penetrating abdominal trauma with injuries of colon & 1 & 0.9 \\
\hline 34. & Penetrating abdominal trauma with injuries of the small intestine & 1 & 0.9 \\
\hline Total & & 111 & 100 \\
\hline
\end{tabular}

Table 2.

The severity of a patient's condition with combined injuries according to the traditional scale and the ISS scale

\begin{tabular}{|l|c|c|c|c|c|}
\hline \multicolumn{2}{|c|}{ Traditional scale } & \multicolumn{2}{c|}{ ISS scale } \\
\hline \multicolumn{1}{|c|}{ Severity of a patient's condition } & $\begin{array}{c}\text { total } \\
(\mathrm{n}=98)\end{array}$ & death rate & ISS range & death rate \\
\hline Satisfactory & $1.0 \%$ & 0 & $<13$ points & $18.4 \% *$ & 0 \\
\hline Moderate severity & $22.4 \%$ & 0 & $14-21$ points & $17.3 \%$ & 0 \\
\hline Severe & $62.2 \%$ & $17.3 \%$ & $22-32$ points & $30.6 \% *$ & $7.1 \% *$ \\
\hline Extremely severe & $8.2 \%$ & $7.1 \%$ & $33-46$ points & $21.4 \% *$ & $12.2 \% *$ \\
\hline Terminal & $6.1 \%$ & $6.1 \%$ & $46-66$ points & $8.2 \% *$ & $7.1 \%$ \\
\hline Lethal & & & 75 points & $4.1 \% *$ & $4.1 \% *$ \\
\hline Total & $100.0 \%$ & $30.5 \%$ & Total & $100.0 \%$ & $30.5 \%$ \\
\hline$P<0.05$
\end{tabular}

$P<0.05$ vs Traditional scale. 
Table 3.

Types of surgical procedures on abdominal organs in FFH victims

\begin{tabular}{|c|c|c|c|}
\hline № & Types of surgical procedures & Abs & $\%$ \\
\hline 1. & Laparoscopic microwave coagulation for hepatic rupture & 2 & 3.4 \\
\hline 2. & Laparoscopic microwave coagulation for splenic rupture & 1 & 1.7 \\
\hline 3. & Laparoscopic appendectomy & 1 & 1.7 \\
\hline 4. & Laparotomy, splenectomy & 17 & 28.8 \\
\hline 5. & Laparotomy, splenectomy + cholecystectomy & 1 & 1.7 \\
\hline 6. & Laparotomy, splenectomy + coagulation / suturing the hepatic rupture & 3 & 5.1 \\
\hline 7. & Laparotomy, splenectomy + suturing the kidney rupture & 1 & 1.7 \\
\hline 8. & Laparotomy, splenectomy + nephrectomy & 2 & 3.4 \\
\hline 9. & Laparotomy, splenectomy + suturing the bladder rupture + suturing the colon rupture & 1 & 1.7 \\
\hline 10. & $\begin{array}{l}\text { Laparotomy, splenectomy }+ \text { coagulation for hepatic rupture }+ \text { suturing the small-intestinal mesentery } \\
\text { rupture }\end{array}$ & 1 & 1.7 \\
\hline 11. & Laparotomy, splenectomy + suturing the colon rupture + suturing the ovarian rupture & 1 & 1.7 \\
\hline 12. & Laparotomy, APC the splenic rupture & 1 & 1.7 \\
\hline 13. & coagulation for splenic capsular tear + coagulation for hepatic rupture & 1 & 1.7 \\
\hline 14. & Laparotomy, suturing and/or coagulation for hepatic rupture & 12 & 20.3 \\
\hline 15. & Laparotomy, suturing the hepatic rupture + removal of retroperitoneal hematoma & 1 & 1.7 \\
\hline 16. & Laparotomy, removal of retroperitoneal hematoma, inspection of the retroperitoneal area & 1 & 1.7 \\
\hline 17. & laparotomy, suturing the small-intestinal/colon mesentery rupture & 2 & 3.4 \\
\hline 18. & Laparotomy, suturing the colon rupture & 1 & 1.7 \\
\hline 19. & Laparotomy, suturing the colon rupture + suturing the ovarian rupture + coagulation for hepatic rupture & 1 & 1.7 \\
\hline 20. & $\begin{array}{l}\text { Laparotomy, coagulation for hepatic rupture, side-to-side entero-entero anastomosis and sigmastoma (due } \\
\text { to co-morbidity, a pelvic tumor) }\end{array}$ & 1 & 1.7 \\
\hline 21. & $\begin{array}{l}\text { Laparotomy, resection of the part of the descending colon with end-to-end colo-colonic anastomosis on the } \\
\text { metallic frame }+ \text { suturing the left kidney rupture with nephrostomy }\end{array}$ & 1 & 1.7 \\
\hline 22. & $\begin{array}{l}\text { Laparotomy, suturing the small-intestinal wall and mesentery rupture, suturing the stomach tear and } \\
\text { sigmoid colon + coagulation for the tears of the left hepatic lobe }\end{array}$ & 1 & 1.7 \\
\hline 23. & Laparotomy, suturing the small intestine rupture & 2 & 3.4 \\
\hline 24. & laparotomy, suturing the hepatic rupture + suturing the small intestine rupture & 1 & 1.7 \\
\hline 25. & Laparotomy, suturing the mesentery defect and deserosation of the small intestine part & 1 & 1.7 \\
\hline 26. & $\begin{array}{l}\text { Laparotomy, suturing the tears of the anterior and posterior walls of the stomach }+ \text { coagulation for the } \\
\text { hepatic rupture }+ \text { suturing the small intestine rupture }\end{array}$ & 1 & 1.7 \\
\hline Total & & 59 & 100 \\
\hline
\end{tabular}

We found differences in the number of patients with varying degrees of severity determined by the traditional grading scale and the ISS scale. These differences were most significant in groups of patients whose condition at admission was determined as moderate, severe, and extremely severe. Thus, the number of patients with combined injuries and a high degree of severity on the ISS scale was 2 times less than the traditional classification, but the number of patients with an extremely severe and terminal condition was 4.15 and 12.35 times greater, respectively. At the same time, the patients admitted with fatal injuries according to the traditional scale were not allocated separately, whereas, $4(4.1 \%)$ patients were given the highest ISS score (75). As shown in Table 2, the severe condition according to the traditional scale was identified in 64 patients and extremely severe and terminal condition in only 8 and 6 patients, respectively. According to the ISS scale, among the admitted patients, the severe condition was determined in 30 patients, extremely severe condition in 21 patients, terminal condition in 8 patients, and a lethal injury in 4 patients. We found a direct correlation between the clinical outcome and severity of the condition at admission. No deaths have been noted among the patients with satisfactory and moderate severity of condition at admission. In the patient group with a severe condition, according to the ISS scale the number of deaths was 2.4 times less than the same group according to the traditional scale. Conversely, in the patient group with the extremely severe and terminal condition according the ISS scale, the number of deaths was 1.72 and 1.16 times greater, respectively.

Active surgical tactics were used in 94 cases: a diagnostic laparoscopy was performed in 35 cases, laparoscopic surgery in 4 patients, conversion to laparotomy in 27 patients, and the initial laparotomy in 28 patients (Table 3). Three (2.7\%) patients with Grade I or II splenic injury were subjected to argon plasma coagulation (APC); splenectomy was performed at Grade III or IV in $27(24.3 \%)$ patients. Electrocoagulation 
and/or APC were performed at Grade II hepatic injury in $34.8 \%$ cases; suturing the liver rupture in combination with coagulation was carried out at Grade III or IV hepatic injury. The ruptures and tears of the hollow organs were sutured in $12(12.8 \%)$ cases; suturing the kidney rupture and nephrostomy was performed on 2 patients, nephrectomy in 2 patients. The diagnostic and treatment procedures in patients with injuries due to FFH with the combined trauma of the abdominal organs were carried out with the participation of surgeons of different specialties. The choice of treatment policy was determined by the severity of a patient's condition, features of injury (the combined or multiple injuries), the severity of the injuries of the abdominal parenchymal organs, and a number of other factors. Overall mortality was $30.5 \%$. Causes of deaths were pneumonia, cerebral edema, and multiple organ failure.

In conclusion, the use of the ISS scale optimizes the analysis of clinical material. Comparative analysis of the assessment of the severity of a patient's condition according to the traditional scale and the ISS scale showed that the ISS scale promotes the active and timely detection of the extremely severe and terminal condition in patients with injuries due to FFH with combined trauma of the abdominal organs. Objective assessment of the severity of trauma and the dominant injury region allows determining the optimal treatment algorithm and predicting the outcome of the injury.

\section{Competing interests}

The authors declare that they have no competing interests.

\section{References}

1. Khadjibaev A Sultanov P. Katatravma: Problems and Perspectives. Bulletin of Emergency Medicine 2013; 4: 8388. [Article in Russian].

2. Dickinson A1, Roberts M, Kumar A, Weaver A, Lockey DJ. Falls from height: injury and mortality. J R Army Med Corps 2012; 158(2):123-7.

3. Abakumov M, Lebedev N, Malyarchuk V. Abdominal injury with an associated trauma. Moscow: Meditsina; 2005. [in Russian].

4. Ermolova A Khubutiya M, Abakumov M. Abdominal trauma. St. Petersburg: Vidar- M; 2010. [in Russian].

5. Alekseev V, Ivanov V, Alekseev S, Vanyukov V. Objective assessment of the severity of injury in victims with splenic injury. Bulletin of Surgery 2013; 1: 50-54. [Article in Russian].

6. Puzanov S, Alishikhov A, Rutenberg G, Bogdanov
D. The usefulness of laparoscopy in traumatic injuries of the abdominal cavity. Endoscopic Surgery 2014; 2: 14-17. [Article in Russian].

7. Longo WE, Baker CC, McMillen MA, Modlin IM, Degutis LC, Zucker KA. Nonoperative management of adult splenic trauma. Criteria for successful outcome. Ann Surg 1989; 210(5):626-9.

8. Bagnenko S. Combined mechanical trauma. St. Petersburg, 2005. [in Russian].

9. Nathens AB, Cryer HG, Fildes J. The American College of Surgeons Trauma Quality Improvement Program. Surg Clin North Am Apr 2012;92(2):441-54

10. Baker SP, O'Neill B, Haddon WJr, Long WB. The Injury Severity Score; a method for describing patients with multiple injuries and evaluating emergency care. J Trauma 1974; 14(3):187-96.

11. Boyd CR, Tolson MA, Copes WS. Evaluating trauma care: the TRISS method.Trauma Score and the Injury Severity Score. J Trauma 1987; 27(4):370-8.

12. Malinin D, Bosco O. Methods of objective assessment of the severity of injuries and their practical application (Guidelines). Volgograd, 2008. [Article in Russian].

13. Brivet F. Scoring system and severe acute pancreatitis. Crit Care Med 2000; 28(8):3124-5.

14. Svetukhin AM, Zviagin AA, Slepnev SIu. Systems of objective evaluation of patients' severity status. Part II. Khirurgiia (Mosk) 2002; 10: 60-9. [Article in Russian].

15. Moreno R, Morais P. Outcome prediction in intensive care: results of prospective, multicentre, Portuguese study. Intensive Care Med 1997; 23(2):177-86.

16. Gumanenko E, Boyarintsev B, Suprun T, et al. Objective evaluation of the severity of injuries. St. Petersburg: Military Medical Academy, 1999. [in Russian].

17. John D. States: The Abbreviated and the Comprehensive Research Injury Scales. In: STAPP Car Crash Journal. 13, Society of Automotive Engineers, Inc., New York 1969, ISSN 1532-8546, S. 282-294, LCCN 67-22372.

18. Committee on Medical Aspects of Automotive Safety. Rating the severity of tissue damage, I. The abbreviated scale. JAMA 1971; 215(2):277-80.

19. Kaida A, Petruk J, Sevcik W, Latoszek K, Ohinmaa A, Jacobs $P$, et al. Investigating the impact of lowering the Injury Severity Score cutoff for major trauma in pediatrics. Acad Emerg Med. 2004;11:513.

20. Aharonson-Daniel L, Giveon A, Stein M; Israel Trauma Group (ITG), Peleg K. Different AIS triplets: different mortality predictions in identical ISS and NISS. J Trauma 2006;61(3):711-7.

21. Moore EE1, Shackford SR, Pachter HL, McAninch JW, Browner BD, Champion HR, et al. Organ injury scaling: spleen, liver, and kidney. J Trauma 1989; 29(12):1664-6. 